\title{
The Complex Spine in Children with Spinal Muscular Atrophy: The Transforaminal Approach-A Transformative Technique
}

\author{
(D) R. Towbin, (D) C. Schaefer, (DR. Kaye, (D)T. Abruzzo, and (DD.J. Aria
}

\begin{abstract}
BACKGROUND AND PURPOSE: Spinal muscular atrophy, a genetic disease resulting in loss of motor function, presents from in utero to adulthood. Depending on progression and secondary scoliosis, spinal stabilization may be necessary. When planning intrathecal access in these patients, spinal anatomy is the most important factor. Therefore, when planning intrathecal nusinersen injections, we subdivided patients with spinal muscular atrophy into simple-versus-complex spine subgroups. Our purpose was to present our experience with our first 42 transforaminal intrathecal nusinersen injections.
\end{abstract}

MATERIALS AND METHODS: We reviewed 31 consecutive patients with spinal muscular atrophy types 1-3 who presented for intrathecal nusinersen injections from March 2017 to September 2018. Nine children had complex spines (ie, spinal instrumentation and/or fusion) and required preprocedural imaging for route planning for subarachnoid space access via transforaminal or cervical approaches.

RESULTS: A total of 164 intrathecal nusinersen injections were performed in 31 children 4-226 months of age, with $100 \%$ technical success in accessing the subarachnoid space. Nine patients with complex spinal anatomy underwent 45 intrathecal nusinersen injections; 42 of 45 procedures were performed via a transforaminal approach with the remaining 3 via cervical techniques. There were no complications.

CONCLUSIONS: Our initial experience has resulted in a protocol-driven approach based on simple or complex spinal anatomy. Patients with simple spines do not need preprocedural imaging or imaging-guided intrathecal nusinersen injections. In contrast, the complex spine subgroup requires preprocedural imaging for route planning and imaging guidance for therapy, with the primary approach being the transforaminal approach for intrathecal nusinersen injections.

ABBREVIATIONS: ITNI = intrathecal nusinersen injection; SMA = spinal muscular atrophy; TF $=$ transforaminal

S pinal muscular atrophy (SMA) is the result of a deletion at exon 7 on the short arm of chromosome 5 and may present from in utero (type 0 ) to adulthood (type 4). Across time, there is a loss of motor neurons in the anterior horn of the spinal cord, resulting in weakness and muscular imbalance. These patients often develop progressive scoliosis. The spinal deformity may further stress individuals with respiratory challenges due to their underlying muscular weakness. Depending on the rapidity of the progression of the spinal curvature and its severity, spinal stabilization with rods and spinal fusion may be necessary. Nusinersen is currently the only FDA-approved drug for the treatment of SMA and is administered intrathecally. The dosing protocol for

Received February 20, 2019; accepted after revision June 17.

From the Department of Radiology (R.T., C.S., R.K., T.A., D.J.A.), Phoenix Children's Hospital, Phoenix, Arizona; and University of Arizona College of Medicine, Phoenix (R.T., C.S., R.K., D.J.A.), Phoenix, Arizona.

Please address correspondence to David J. Aria, MD, MBA, Phoenix Children's Hospital, 1919 E Thomas Rd, Department of Radiology, Phoenix, AZ 85016; e-mail: daria@phoenixchildrens.com

http://dx.doi.org/10.3174/ajnr.A6131 nusinersen administration is divided into induction and maintenance phases. The induction phase consists of 3 doses administered 14 days apart followed by a fourth dose given 30 days later. The maintenance phase lasts indefinitely, with dose administration at 4-month intervals. Children with types 1 and 2 SMA may not require spine instrumentation until they are about 6 years of age. ${ }^{1}$ However, older individuals often require rods and/or spinal fusion.

When planning the route to the intrathecal space in patients with SMA, it seems that the status of the spine is the most important factor. Therefore, instead of using the SMA typing system (types $0-4$ ) to plan the procedure, we subdivide patients into simple and complex spine subgroups to plan their therapeutic approach. Clinical history, physical examination findings, and radiographs, if available, are reviewed. For procedural planning, spines of individuals, regardless of age or SMA type, who do not have rods or spinal fusion have been termed "simple spines." Spines of those with instrumentation (ie, spinal rods) and/or fusion are deemed "complex spines". ${ }^{2}$ 


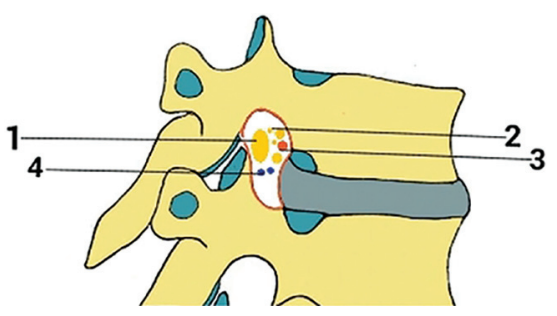

FIG 1. A view illustrating some of the components of the thoracic intervertebral foramina including the following: 1) segmental spinal nerve/dorsal root ganglion, 2) sinuvertebral nerves and rami communicantes, 3) spinal branch of segmental arteries, and 4) intervertebral veins. $^{3}$

The purpose of this article was to present our experience with our 42 transforaminal (TF) intrathecal nusinersen injections (ITNIs). To our knowledge, this is the largest reported series of TF nusinersen therapy for SMA.

\section{MATERIALS AND METHODS}

Our entire patient population from the initiation of intrathecal therapy with nusinersen was examined. We reviewed all patients with SMA who were referred for ITNIs between March 2017 and September 2018 at Phoenix Children's Hospital. Thirty-one children with SMA were treated. Of these, 9 children had complex spines and were the focus of this presentation. Patients with complex spines required preprocedural spinal radiographs and lumbar spine CT and imaging-guided ITNIs. 3D reformatting was performed as needed. All ITNIs were performed in an interventional radiology suite equipped with an AlluraClarity FD Biplane Angiography System (Philips Healthcare, Best, the Netherlands), regardless of whether fluoroscopic or conebeam CT guidance (Allura Xper FD system; Philips Healthcare) was used. Four pediatric interventionalists, with experience ranging from 4 to 38 years (4, 10,26 , and 38 years, respectively) performed all procedures. Fluoroscopy times were recorded when fluoroscopic guidance was used.

All procedures were performed using a sterile technique with needle selection dependent on patient body habitus and operator preference. The needles of choice were 22-ga, ranging from 3.5 to 7 inches and, in almost all instances, were the Quincke type. On occasion, Whitacre needles (Becton, Dickinson, and Company) were used, particularly if there was a history of a spinal headache. In obese patients, a coaxial technique may be used to minimize needle tracking. Coaxial needle insertion consisted of an 18-ga 10-cm Chiba Biopsy needle (Cook Medical, Bloomington, Indiana) as a guide with an inner 22-ga 7-inch lumbar puncture needle. Needle tip position was confirmed by the acquisition of CSF. All children who received ITNIs were followed clinically by their referring neurologist and physical medicine and rehabilitation teams. All untoward effects were identified and reviewed.

\section{Transforaminal Puncture Technique}

Before considering the TF approach, one must understand the anatomy of the neural foraminal space. The neurovascular bundle is located within the upper half of the neural foraminal space. Therefore, the lower half of the neural foraminal space is targeted, with the posterior-inferior quadrant the ideal location (Fig 1). ${ }^{3}$ The lumbar entry level and sidedness are selected after reviewing the lumbar spine CT. When needed, a 3D reconstruction is used to better understand the pathologic anatomy and anticipated needle course.

In all cases, the procedure was performed with the patient under general anesthesia. The child is placed in the decubitus position with the convex side of the scoliotic curvature at the planned lumbar entry level directed toward the ceiling. This positioning allows the ostial plane of the neural foramina to be perpendicular to the radiation beam, optimizing visualization of the neural foraminal space. In children with complex spines, it is commonly not feasible to position the entire spine in an ideal position visualizing multiple neural foraminal levels. Therefore, we focus on positioning only the previously determined target level in the projection maximizing the visibility of the target neural foramina. Also, many of these children have severe osteopenia, making it challenging to distinguish the vertebral body from superimposed bowel gas. The needle tip is positioned over the posterior-inferior quadrant of the neural foramina. When the operator is looking down the hub of the puncture needle, he or she can be confident that the needle angle is appropriate. A 5- or 7-inch needle is used due to the longer distance to the intrathecal space from a paraspinal approach. In our early experience, conebeam CT and XperCT navigation (Philips Healthcare) and guidance (Allura Xper FD System) were used to assist needle placement (Fig 2). ${ }^{4}$ As we became more comfortable and experienced with the TF approach, we substituted fluoroscopic guidance as our first option (Fig 3).

\section{Cervical Puncture Technique}

When a cervical approach is considered, additional preprocedural imaging is performed. In this subgroup, limited cervical MR imaging is obtained for assessing the CSF space posterior to the spinal cord. Depending on operator preference, the patient may be positioned in the prone, supine, or decubitus position. It is our preference to use the decubitus position because it maximizes access to the craniocervical junction region, especially in smaller children. The preferred skin-entry site is at C1-2 with the needle positioned about midway between the flare point ${ }^{5}$ anteriorly that approximates the posterior border or the spinal cord and the spinal laminar line posteriorly that approximates the margin of the subarachnoid space (Fig 4). A 3.5-inch needle is used, and under continuous biplane fluoroscopic guidance, the needle tip is positioned in the midline, confirmed on the frontal projection. If $\mathrm{C} 1-2$ access cannot be achieved, alternative approaches include the $\mathrm{C} 1$ cisterna magna and suboccipital approaches. Successful needle position is confirmed by acquisition of CSF or contrast injection.

All technical details, including fluoroscopy time, anesthetic type, procedural details, and untoward events, were documented.

\section{RESULTS}

A total of 164 ITNIs were performed in 31 children with simple and complex spines, 4-226 months of age, with a mean age of 97.9 months at the time of initial therapy. Of these, 9 children had complex spines and underwent a total of 45 ITNIs. There was 100\% technical success in accessing the subarachnoid space in a single session.

Each child had 4-6 procedures with a mean of 5.0 injections. Of these 9 patients, there were 5 girls and 4 boys who ranged in age from 119 to 216 months, with a mean age of 173.8 months. The subgroup 

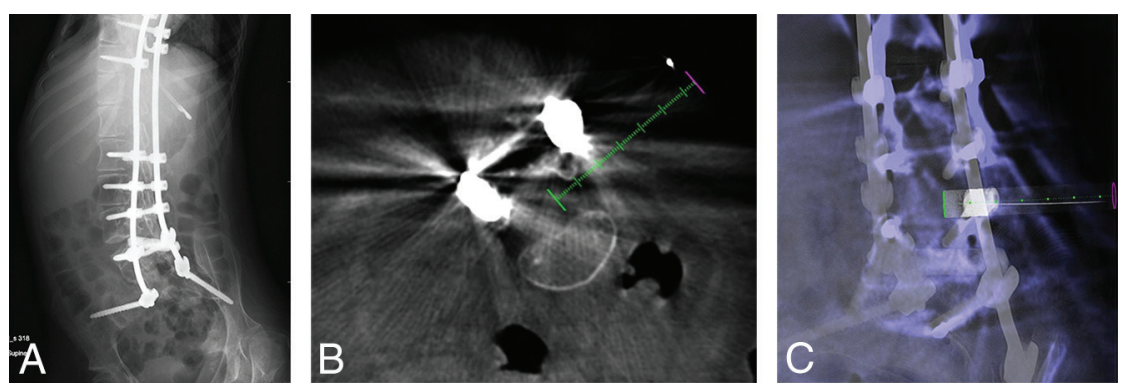

FIG 2. A, A 14-year-old girl with SMA type 2 and an abdominal radiograph demonstrating thoracolumbar spinal rods extending to the sacrum with posterior spinal osseous fusion. $B$, Conebeam CT using XperCT guidance software (Philips Healthcare) (green dashed line) to access the thecal sac via a right L3-L4 transforaminal approach. C, XperCT fluoroscopic overlay "progress" view used to determine needle tip position depth in the thecal sac.
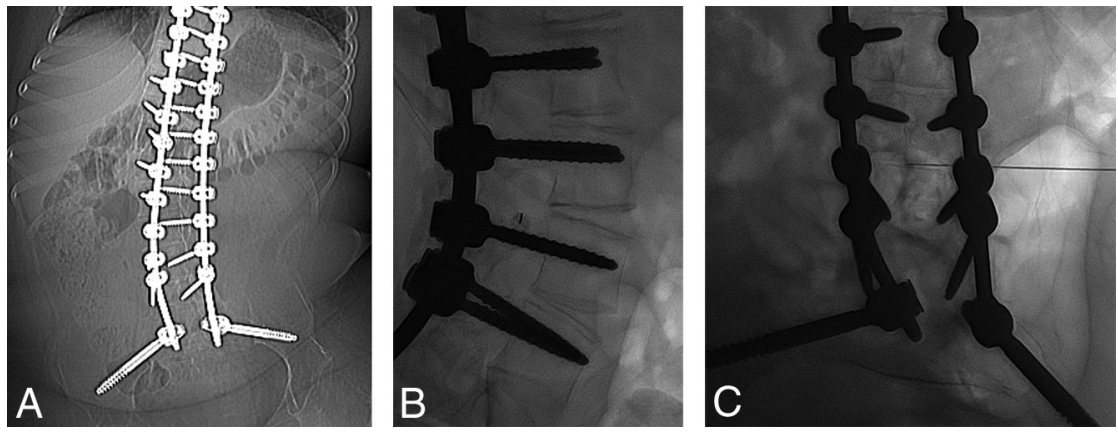

FIG 3. A, A 15-year-old boy with SMA type 3. Frontal abdominal radiograph demonstrates diffuse spinal fusion extending to the sacrum. $B$, With the patient in the left lateral decubitus position, a true-lateral radiograph at the L3-L4 level demonstrates the spinal needle positioned over the posteroinferior aspect of the right neural foramina. $C$, With the orthogonal view, the spinal needle tip is centered in the thecal sac.
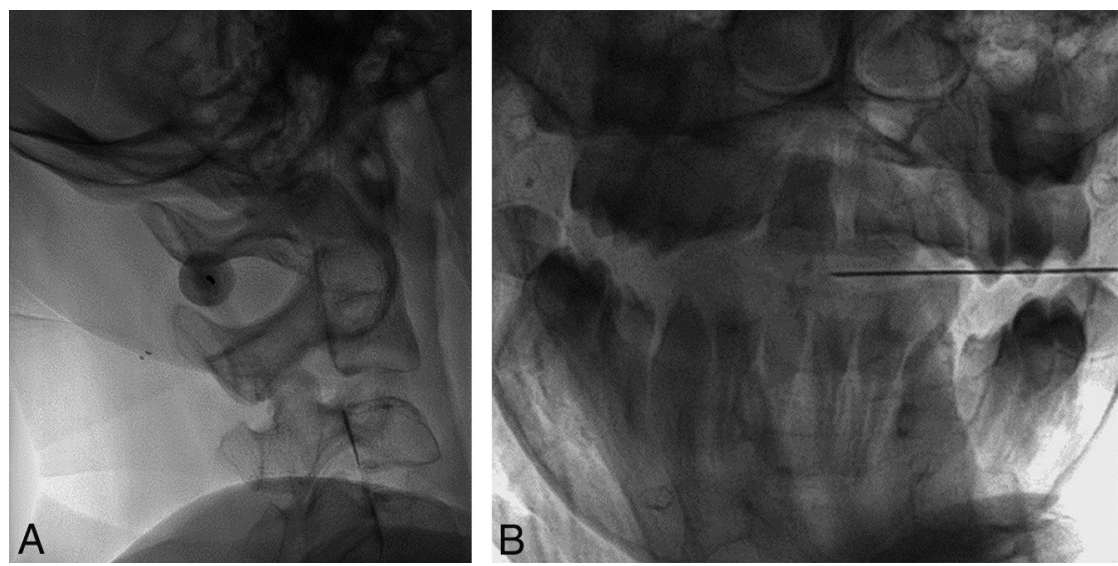

FIG 4. A, A 14-year-old boy with SMA type 3 and diffuse osseous spinal fusion. With the patient in lateral decubitus position, lateral fluoroscopic image demonstrates the spinal needle superimposed over the $\mathrm{Cl}-\mathrm{C} 2$ interspace. $\mathrm{B}$, The orthogonal view is used for monitoring progress of the needle tip position to midline.

was composed of 6 children with type 2 SMAs who had an age range of 119-201 months and mean age of 175.2 months, and 3 children with type 3 SMA with an age range of 168-226 months and a mean of 193.1 months. All 9 patients had both spinal rods and long-segment spinal fusion.

All 45 procedures were performed with the patient under general anesthesia, though the anesthesiologist's method of ventilation varied. Of the 45 ITNIs, a laryngeal mask airway was used in 40 cases; endotracheal intubation, in 2; and mask ventilation, in 3.
Almost all children had their ITNIs performed in the decubitus position. Fourteen procedures were performed with the patient in the right lateral decubitus position; 28, in left lateral decubitus; and 3, with the patient prone for navigation and XperCT guidance. Cervical spine puncture was used in 3 procedures, all performed with the patient in the left decubitus position. The skin-entry site levels varied minimally with 1 at the $\mathrm{C} 1$ cisterna magna, 2 at $\mathrm{C} 1-2$, one at $\mathrm{L} 2-3$, thirtyseven at L3-4, and 4 at L4-5. As experience was gained with the TF approach, the cervical approach was abandoned. In our practice, the first option is the TF approach with cervical approaches considered if the TF approach fails.

Forty-one of the 45 procedures used a 5 - or 7 -inch needle. The remaining 4 injections used a 3.5-inch needle, of which 3 were for cervical approaches. For fluoroscopically guided TF access, fluoroscopy times ranged from 48 seconds to 4 minutes 54 seconds with a mean of 2.0 minutes. Conebeam CT-guided TF injections averaged 4 minutes 6 seconds, with the C1-2 puncture taking 4 minutes $54 \mathrm{sec}$ onds and the $\mathrm{C} 1$ cisterna magna puncture taking 14 minutes 30 seconds, for a mean of 8 minutes 6 seconds for both cervical routes. Room times varied, with a range of 27-89 minutes, with a mean time of 50 minutes 18 seconds. There were no immediate or reported shortterm complications.

\section{DISCUSSION}

In reviewing our data, we strongly believe that our $100 \%$ technical success rate in obtaining intrathecal access was aided by preprocedural imaging. Because all children with complex spinal anatomy underwent lumbar CT for route planning of ITNIs, we were able to identify patent access routes to the intrathecal space before procedural scheduling. In our experience, the diagnostic technique of choice is lumbar CT, with $3 \mathrm{D}$ reconstructions as needed, because it allows assessment of TF (and even interspinous) approaches at all lumbar levels. Once the intrathecal access route is identified, patient positioning needs can be relayed to the anesthesiologist involved in the procedure, thereby reducing the need for repositioning and extended room and general anesthesia times. Also, if needed, the lumbar CT data can be transferred to the CT navigation system in the interventional radiology suite for imaging guidance. 


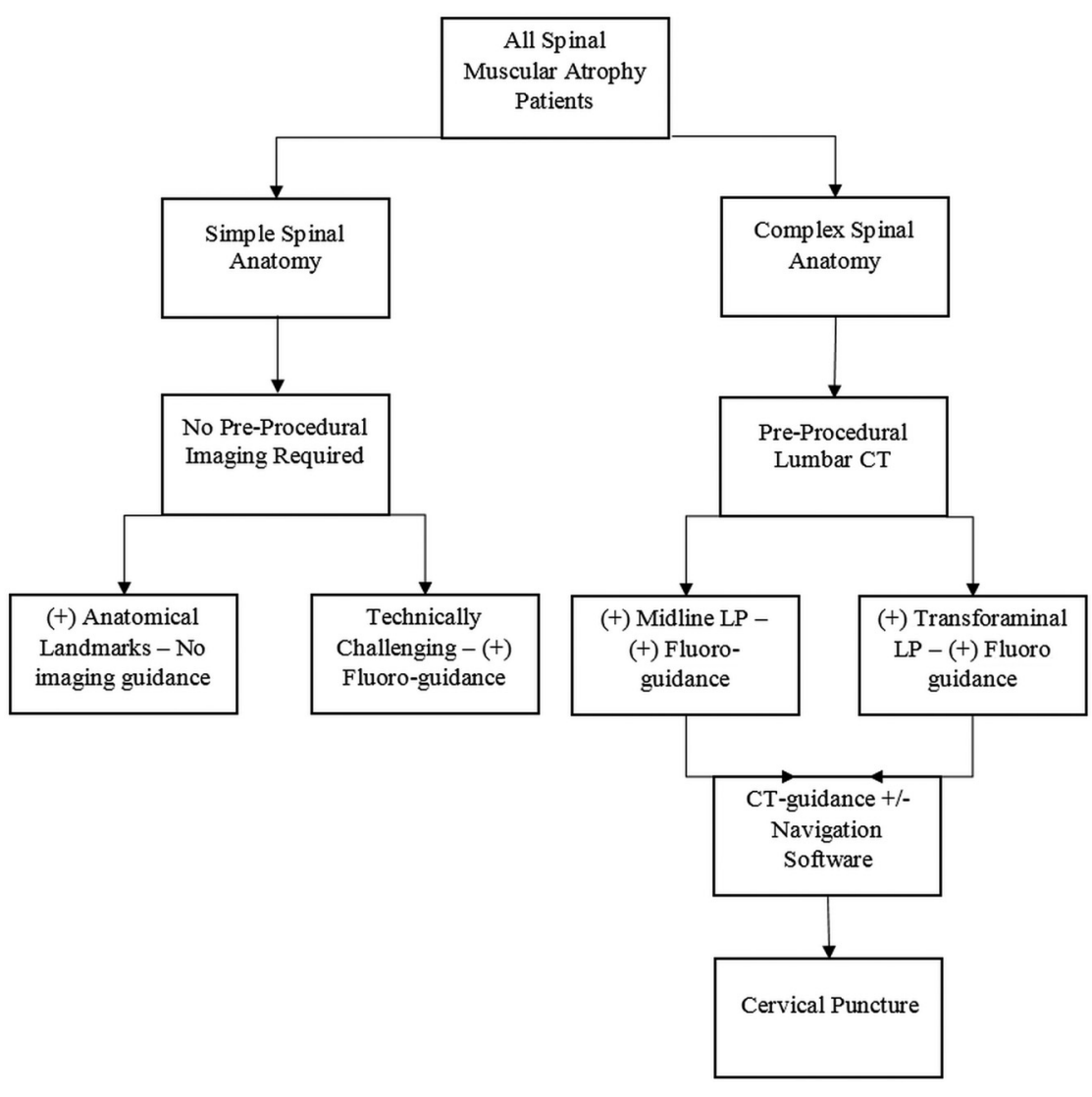

FIG 5. An algorithmic approach to ITNIs in patients with spinal muscular atrophy.

in case fluoroscopic guidance should fail. However, an interventional radiology suite with conebeam CT navigational guidance available is helpful in 2 ways: First, the interventional radiology suite serves as a central location where all necessary guidance tools are available, avoiding patient transportation and traveling concerns; and second, it offers the most sophisticated setting for maximizing the likelihood for technical success.

Historically, the TF approach has been used for epidural steroid injections $^{7}$ and for myelography, with an excellent safety and technical success history, specifically when image guidance is used. ${ }^{6}$ The primary risks of the TF approach include nerve and vascular injury, potential bleeding, and spinal cord injury. Transgression of the kidney is possible, but preprocedural lumbar CT helps plan a route that avoids the kidney, as does patient positioning with the convex side of the spinal curvature toward the ceiling. Targeting the lower half of the neural foramina is key to avoiding neurovascular injury. In our experience, no complications occurred, while prior

Although all of our TF ITNIs were performed with the patient under general anesthesia, the method of ventilation varied. Children with SMA commonly have compromised pulmonary function due to scoliosis, spinal fusion, and weakened chest wall musculature. Therefore, minimizing respiratory strain on the patient with SMA is important in their management. Because our preferred position for the TF approach is left- or right-sided lateral decubitus, there is a reduced need for endotracheal intubation compared with the prone position. In our population, general anesthesia via an endotracheal tube was used in only 2 of 42 TF procedures. The avoidance of endotracheal intubation leads to a shorter postprocedural recovery time compared with the laryngeal mask airway or mask ventilation approaches and a decreased risk of prolonged intubation, which is significantly higher in patients with SMA than in the general population.

As our experience with the TF approach to ITNIs evolved, the use of CT-guided navigation ceased. In fact, the use of CT guidance was limited to 3 early TF ITNIs compared with 39 subsequent fluoroscopically guided TF ITNIs. Previously, the literature almost exclusively reported using CT guidance for the TF approach. ${ }^{4,6}$ Despite our transition to a rotating fluoroscopic C-arm for guidance, given the advanced technology in our interventional radiology suite with both fluoroscopic and CT navigation guidance available, we suggest the interventional radiology suite as the ideal setting for the procedure. At practices or institutions where CT guidance is not available in the interventional radiology suite, a diagnostic CT scanner is a viable second option for imaging guidance and should be reserved before beginning the procedure reports indicated occasional minor complications. ${ }^{4,6}$ Collectively, all TF ITNIs were technically successful.

When caring for pediatric patients specifically, being aware and considerate of fluoroscopy time and radiation exposure is a high priority. Most interesting, our experience with the fluoroscopically guided TF approach revealed an unexpected observation. Although we anticipated that our fluoroscopically guided TF fluoroscopy times (mean, 2 minutes) would compare favorably with the cervical (mean, 8 minutes 6 seconds) and CT-guided TF (mean, 4 minutes 6 seconds) approaches, we did not expect the times to be similar to those that we commonly see with the midline interspinous lumbar approach (mean, 2 minutes). Given the longer trajectory to the intrathecal space via the TF approach, as confirmed with the predominant use of 5- or 7-inch spinal needles, the probability of needle deflection increases and may increase fluoroscopy time or decrease the success of intrathecal access. These untoward effects may have been minimized with the use of a coaxial needle technique as discussed previously.

Last, in 2 children with SMA with complex spines, 3 cervical punctures were performed because we were not yet using the TF approach. Although cervical approaches were successful without complications, the TF approach is preferred because of the lower risk profile and the extended fluoroscopy times encountered with the cervical routes. In both patients, the TF approach was subsequently used for future ITNIs.

The limitations of this study include its small number of transforaminal injections and its lack of comparison with other methods and techniques. A variety of alternative needle approaches to 
the subarachnoid space has been described, including transforaminal, interlaminar, cervical, and suboccipital into the cisterna magna, sacral canal, and sacral foramina. In addition, there are other considerations such as surgical or percutaneous access with device implantation and/or creation of bone windows. In our series, we explored the cervical and TF approaches but did not use implanted devices or bone windows.

\section{CONCLUSIONS}

Our initial experience has led us to create a protocol-driven approach to guide our technical choices based on the patient's spinal anatomy, whether simple or complex. Patients with simple spines without instrumentation generally do not need preprocedural imaging to assess spinal anatomy, and most do not need imaging guidance. In contrast, the complex spine group requires preprocedural imaging and imaging guidance with the primary approach being the TF approach. With the evolution of the interventionalists' experience, fluoroscopically guided transforaminal ITNIs should be the preferred method. Figure 5 presents our algorithmic approach to these patients.

Disclosures: Richard Towbin—UNRELATED: Expert Testimony: medicolegal, primarily pediatric brain injury; Royalties: Halyard Health.

\section{REFERENCES}

1. Lenhart RL, Youlo A, Schroth Mk, et al. Radiographic and respiratory effects of growing rods in children with spinal muscular atrophy. J Pediatr Otrthop 2017;37:e500-04 CrossRef Medline

2. Mousa M, Aria D, Schaefer C, et al. A comprehensive institutional overview of intrathecal nusinersen injections for spinal muscular atrophy. Pediatr Radiol 2018;48:1797-1805 CrossRef Medline

3. Gkasdaris G, Tripsianis G, Kotopoulos K, et al. Clinical anatomy and significance of the thoracic intervertebral foramen: a cadaveric study and review of the literature. J Craniovertebr Junction Spine 2016;7: 228-35 CrossRef Medline

4. Weaver JJ, Natarajan N, Shaw DW, et al. Transforaminal intrathecal delivery of nusinersen using conebeam computed tomography for children with spinal muscular atrophy and extensive surgical instrumentation: early results of technical success and safety. Pediatr Radiol 2018;48:392-97 CrossRef Medline

5. Peckham ME, Shah LM, Tsai AC, et al. C1 posterior arch flare point: a useful landmark for fluoroscopically guided C1-2 puncture. AJNR Am J Neuroradiol 2018;39:1562-67 CrossRef Medline

6. Nascene DR, Ozutemiz C, Estby H, et al. Transforaminal lumbar puncture: an alternative technique in patients with challenging access. AJNR Am J Neuroradiol 2018;39:986-91 CrossRef Medline

7. Botwin KP, Gruber RD, Bouchlas CG, et al. Complications of fluoroscopically guided transforaminal lumbar epidural injections. Arch Phys Med Rehabil 2000;81:1045-50 CrossRef Medline 